\title{
$5(R)-(I$-真氧基)-2(5H)-呋喃酮与溴代丙二酸二乙酯的反应
}

\author{
康海霞 $^{a}$ 白 静 ${ }^{b}$ 郗振涛 ${ }^{a}$ 张曼曼 ${ }^{a}$ 傅玉琴 $*, a$ 邹大鹏 $*, b$ \\ ( ${ }^{a}$ 洛阳师范学院化学化工学院 洛阳 471022) \\ ( ${ }^{b}$ 郑州大学化学与分子工程学院 郑州 450001)
}

\begin{abstract}
摘要 研究了 $5(R)-\left(l\right.$-真氧基)-2 $(5 H)$-呋喃酮与溴代丙二酸二乙酯在无水 $\mathrm{K}_{2} \mathrm{CO}_{3}$ 和相转移催化剂四丁基溴化铵(TBAB) 存在下, 以乙腈为溶剂, 在 $80{ }^{\circ} \mathrm{C}$ 下的反应及产物结构特征. 在上述条件下得到了预期的具有两个乙酯基的手性环丙烷 /丁内酯衍生物 3, 同时还得到了含有一个乙酯基的手性环丙烷/丁内酯化合物 $\mathbf{5}$ 以及少量的含溴产物 $\mathbf{6}$. 通过对产物结 构分析，提出了产物形成的可能机理.
\end{abstract}

关键词 2(5H)-呋喃酮; 环丙烷/丁内酯衍生物; 手性; 合成; 晶体结构

\section{Reaction of 5(R)-(I-Menthoxy)-2(5H)-furanone with Diethyl Bromomalonate}

\author{
Kang, Haixia ${ }^{a} \quad$ Bai, Jing $^{b} \quad \mathrm{Xi}_{\text {, Zhentao }}{ }^{a} \quad$ Zhang, Manman $^{a}$ \\ $\mathrm{Fu}$, Yuqin ${ }^{*, a} \quad$ Zou, Dapeng ${ }^{*, b}$ \\ $\left({ }^{a}\right.$ College of Chemistry and Chemical Engineering, Luoyang Normal University, Luoyang 471022) \\ ( ${ }^{b}$ College of Chemistry and Molecular Engineering, Zhengzhou University, Zhengzhou 450001)
}

\begin{abstract}
The reaction of 5(R)-(l-menthoxy)-2(5H)-furanone and diethyl bromomalonate was investigated in the presence of anhydrous $\mathrm{K}_{2} \mathrm{CO}_{3}$ and phase transfer catalyst tetrabutylammonium bromide (TBAB) using $\mathrm{CH}_{3} \mathrm{CN}$ as solvent at $80{ }^{\circ} \mathrm{C}$. In the above condition, the expected novel chiral cyclopropane/butyrolactone derivative $\mathbf{3}$ with two ethyl ester groups was obtained, and at the same time, another chiral cycloproane/butyrolactone 5 bearing only one ethyl ester group, and trace amount of bromo-substitued compound $\mathbf{6}$ were also obtained. The possible mechanism was proposed based on the analysis of the products.
\end{abstract}

Keywords 2(5H)-furanone; cyclopropane/butyrolactone derivative; chiral; synthesis; crystal structure

$\gamma$-丁内酯结构单元广泛存在于天然产物中, 许多具 有该类结构单元的化合物具有抗病毒、抗菌、抗炎、抗 癌等生理活性 ${ }^{[1 \sim 4]}$, 因此该类化合物的合成一直备受研 究者的关注 ${ }^{[59]}$. 糠醛在光敏剂玫瑰红(rose bengal)存在 下, 经氧气氧化可得 5-羟基-2(5H)-呋喃酮, 进一步与薄 荷醇缩醛化可方便地得到具有 $\gamma$-丁烯内酯结构单元的 手性源 $5(R)$-( $\left(l\right.$-真氧基)-2(5H)-呋喃酮 $(1 \text {, 图 1 })^{[10,11]}$. 具有 多个反应中心的手性源 1 及其 3-卤代、3,4-二卤代衍生

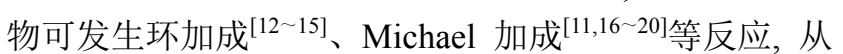
而构建具有复杂结构的多官能团 $\gamma$-丁内酯衍生物.

含有环丙烷结构单元的化合物不仅具有较强的生 理活性 ${ }^{[21 ~ 24]}$, 且其三元环具有刚性的平面结构、特殊的
键角、可带有多个手性中心、易于发生亲电开环反应等 诸多特点，使环丙烷类化合物的构建成为有机化学领域 的研究热点. 构建环丙烷结构的方法很多, 其中以烯烃 为原料的加成反应是构建环丙烷化合物的主要途径之 一. 手性源 1 可以通过加成反应构建环丙烷结构, 从而 形成环丙烷/丁内酯衍生物, 从理论上具有生理活性优 化叠加的意义. 本课题组曾以 $5(R)-(l$-蓋氧基) -3 -溴$2(5 H)$-呋喃酮与含氧、碳、氮等亲核试剂之间的串联双 Michael 加成/分子内亲核取代反应构筑了一系列螺环/ 环丙烷/丁内酯化合物 $\mathbf{2}^{[25 \sim 28]}$ (图 1). Maestro 等 ${ }^{[29]}$ 研究了 $5(R)-(l$-真氧基)-3-溴-2 $(5 H)$-呋喃酮和丙二酸二乙酯在氢 化钠存在下的反应，并同时得到了与两分子底物反应形

*E-mail: lyfuyuqin@126.com

Received January 29, 2016; revised March 22, 2016; published online May 3, 2016.

Project supported by the Foundation of Science and Technology of Henan Province (No.122300410265) and the Fostering Foundation of Luoyang Normal University in 2013 (No. 2013-PYJJ-006).

河南省科技发展计划(No. 122300410265)及洛阳师范学院 2013 年培育基金(No. 2013-PYJJ-006)资助项目. 
成的螺环/环丙烷/丁内酯衍生物 2 和与一分子底物反应 生成的环丙烷/丁内酯化合物 3 (图 1)两种产物. 以上研 究均是以手性源 1 的 3-溴代衍生物为底物合成环丙烷衍 生物, 由手性源 1 直接构建环丙烷结构的方法仅见 Oikawa 等 ${ }^{[30]}$ 报道的和重氮甲烷在 $-40{ }^{\circ} \mathrm{C}$ 进行的 1,3 -偶 极环加成反应, 作者通过光化学反应脱氮, 得到了环丙 烷/丁内酯化合物 4(图 1).

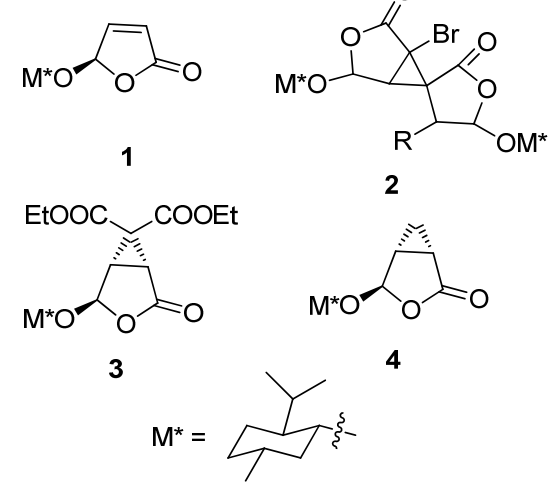

图 1 化合物 $1 \sim 4$ 的结构

Figure 1 Structures of compounds $1 \sim 4$

与丙二酸二乙酯相比, $\alpha$-溴代丙二酸二乙酯的 $\alpha-\mathrm{H}$ 具有更强的酸性, 在碱性条件下更容易形成碳负离子, 同时溴又是易于离去的基团，我们设计以 $\alpha$-溴代丙二酸 二乙酯在弱碱性条件下与 $5(R)$-( $l$-蒀氧基)-2(5H)-呋喃酮 (1) 直接反应, 也可能形成环丙烷/丁内酯衍生物 3 (Scheme 1), 其反应机理可能是串联的 Michael 加成/分 子内亲核取代反应, 或者卡宾反应. 实验结果表明, 该 反应不仅得到了预期的手性环丙烷/丁内酯衍生物 3, 同 时还得到了另一环丙烷/丁内酯衍生物 5 以及少量的含 溴化合物 $\mathbf{6}$, 其中 3 和 5 的绝对构型首次通过 $\mathrm{X}$ 射线单 晶衍射确认.

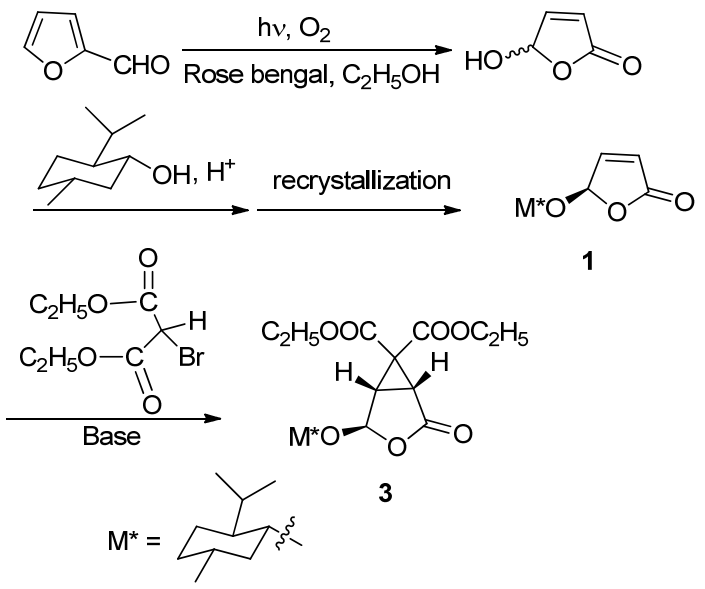

图式 1 目标化合物 $\mathbf{3}$ 的合成路线设计

Scheme 1 Synthetic route of the target compound 3

\section{1 结果与讨论}

\section{1 目标化合物的合成}

Maestro 等 ${ }^{[29]}$ 以 $5(R)$-( $l$-蒀氧基)-3-溴-2(5H)-呋喃酮 和丙二酸二乙酯合成化合物 3 的反应在二甲基甲酰胺 (DMF)中进行, 以氢化钠作碱. 由于 $\alpha$-溴代丙二酸二乙 酯的 $\alpha-\mathrm{H}$ 比丙二酸二乙酯具有更强的酸性，因此，我们 选择了弱碱碳酸钾，以乙腈为溶剂，四丁基溴化铵 $(\mathrm{TBAB})$ 作相转移催化剂, 在外浴 $80{ }^{\circ} \mathrm{C}$ 条件下反应. 采 用碳酸钾促进反应的优势一是污染小，二是反应完毕只 须过滤即可除去相转移催化剂 TBAB 和弱碱碳酸钾，操 作简便. 乙腈不仅提供了一个与 DMF 相似的强极性溶 剂环境, 利于加成反应的进行, 而且沸点比 DMF 低, 反 应完成后可以直接蒸馏去除.

实验在 $80{ }^{\circ} \mathrm{C}$ 油浴中加热进行，待原料基本消失后， 终止反应，过滤，滤液浓缩后用硅胶进行柱层析分离得 到了目标产物环丙烷-丁内酯衍生物 3 (Scheme 2), 同时 还得到了环丙烷-丁内酯化合物 $\mathbf{5}$ 和极少量化合物 $\mathbf{6}$, 化 合物 5 比预期产物 3 少了一个酯基，而化合物 $\mathbf{6}$ 还有溴， 没有形成三元环，且只有一个酯基.

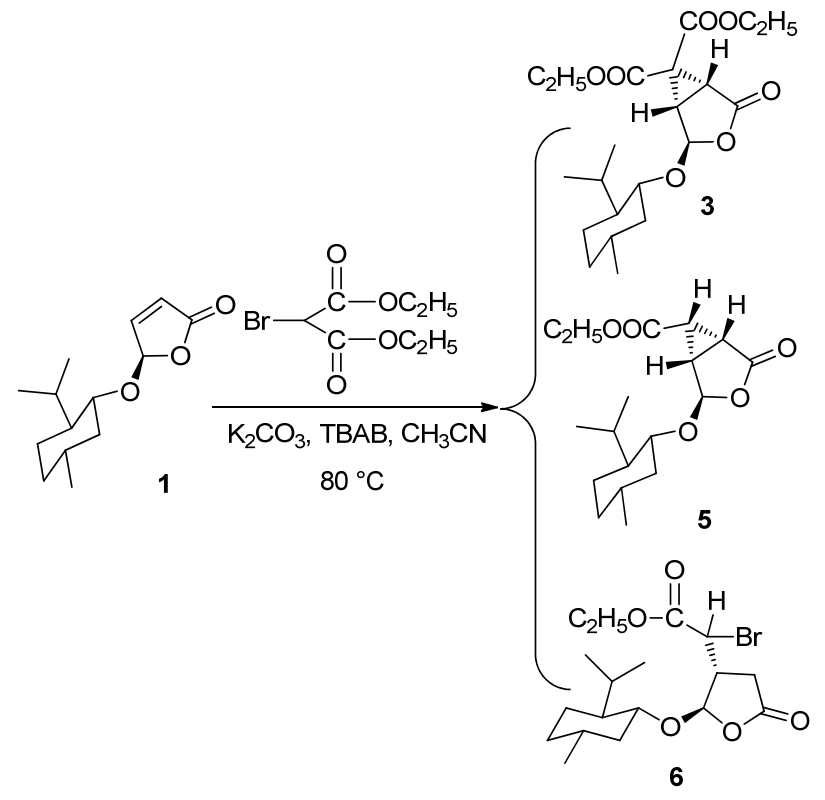

图式 2 化合物 3,5 和 6 的合成

Scheme 2 Synthesis of compounds 3, 5 and $\mathbf{6}$

\section{2 反应机理探讨}

关于化合物 3 中三元环结构的形成，其可能的反应 机理有两种: 一是 Michael 加成/分子内亲核取代机理 (Scheme 3), 即溴代丙二酸二乙酯在碱的作用下, 形成 碳负离子中间体，然后与一分子 $5(R)$-( $l$-蓝氧基)- $2(5 H)$ 呋喃酮发生 Michael 加成形成负离子中间体 7, 继而发 生分子内亲核取代反应脱去溴，形成环丙烷结构，得到 
化合物 3; 二是卡宾机理, 即在碱性条件下溴代丙二酸 二乙酯发生 $\alpha$-消除形成卡宾, 然后与手性源发生加成反 应形成三元环. 由于在反应中得到了少量的含溴产物 $\mathbf{6}$, 化合物 6 可以看成是手性源 1 与溴代丙二酸二乙酯发生 Michael 加成得到中间产物 8 后再脱酯基而得(Scheme 3). 虽然从实验结果无法确定反应中是否有卡宾形成, 但从副产物 6 的形成我们可以推测三元环结构的形成一 定存在 Michael 加成/分子内亲核取代过程.

化合物 5 是化合物 3 的脱酯基产物, 我们推测化合 物 3 的酯基脱除过程按 Scheme 4 进行, 即在碳酸盐的作 用下, 经过 $\mathrm{HCO}_{3}^{-}$对酯羰基的加成、脱 $\mathrm{EtO}^{-}$、两次脱 $\mathrm{CO}_{2}$, 最终得到脱酯基产物 $\mathbf{5}$. 若假设是先脱酯基再发 生加成反应, 则用溴代乙酸乙酯代替溴代丙二酸二乙酯 应得到同样的产物, 但实验证实得不到化合物 5. 理论 上, 化合物 3 脱酯基后, 与酯基相连的 $\mathrm{C}$ 变成手性碳, 化合物 5 中三元环上的三个 $\mathrm{H}$ 应存在两种构型, 即与酯
基相连的碳上的 $\mathrm{H}$ 与桥头碳上的两个 $\mathrm{H}$ 处于反式或顺 式，即化合物 5 应是一对差向异构体. 从氢谱图上可以 看到乙基上的 $\mathrm{CH}_{2}$ 峰形比较特别, 不是理论上的四重 峰, 而表现出对称的多重峰, 我们曾推测这有可能是因 为差向异构体结构太相似而引起的, 其它 $\mathrm{H}$ 的峰形以及 碳谱又特别简单，没有差向异构体的特征. 而且，进一 步的单晶结构测试发现 5 中的三元环是顺式结构(图 3). 综合分析，我们认为产物 5 应是单一构型化合物. 而同 样失去一个酯基的化合物 $\mathbf{6}$ 的形成过程与 5 相似. 化合 物 6 中与 $\mathrm{Br}$ 相连的 $\mathrm{C}$ 原子也是手性碳, 但从氢谱和碳 谱上没有看到差向异构体的特征, 故推测其是单一构型 为主的化合物. 在这个反应中, 选择性脱酯基的原因还 有待于进一步研究.

\section{3 化合物 3 和 5 的单晶结构描述}

化合物 3 的 $X$ 射线单晶衍射数据如下: $\mathrm{C}_{21} \mathrm{H}_{32} \mathrm{O}_{7}$, 属 于单斜晶系, $C 2$ 空间群. 晶胞参数 $a=22.517(3) \AA, b=$

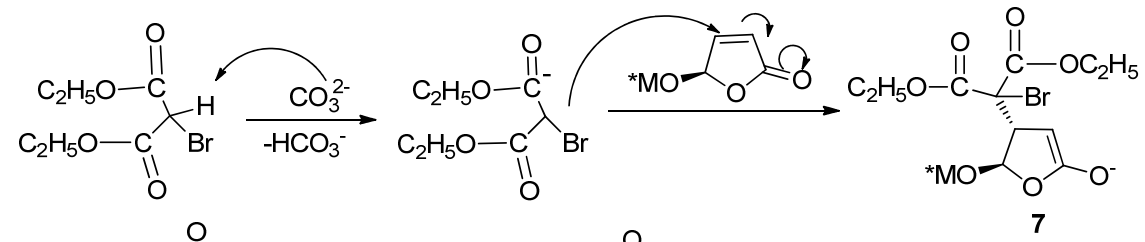

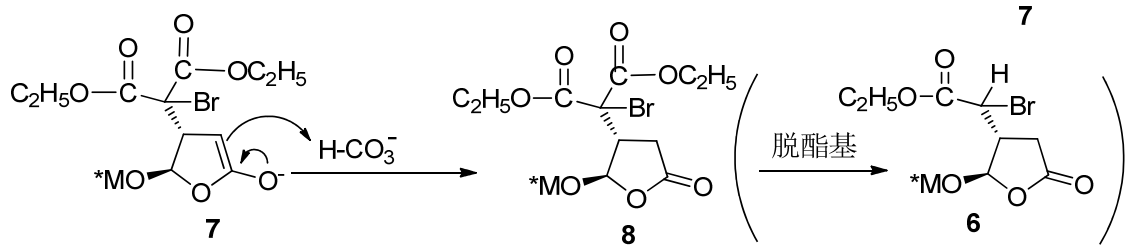

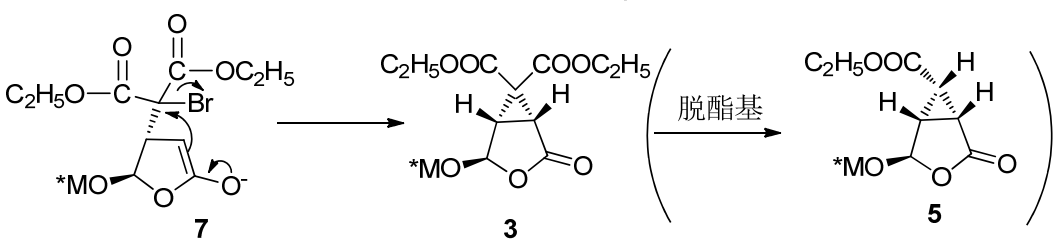

图式 3 化合物 3 环丙烷结构形成的反应机理

Scheme 3 Proposed mechanism for the formation of cyclopropane structure in compound 3

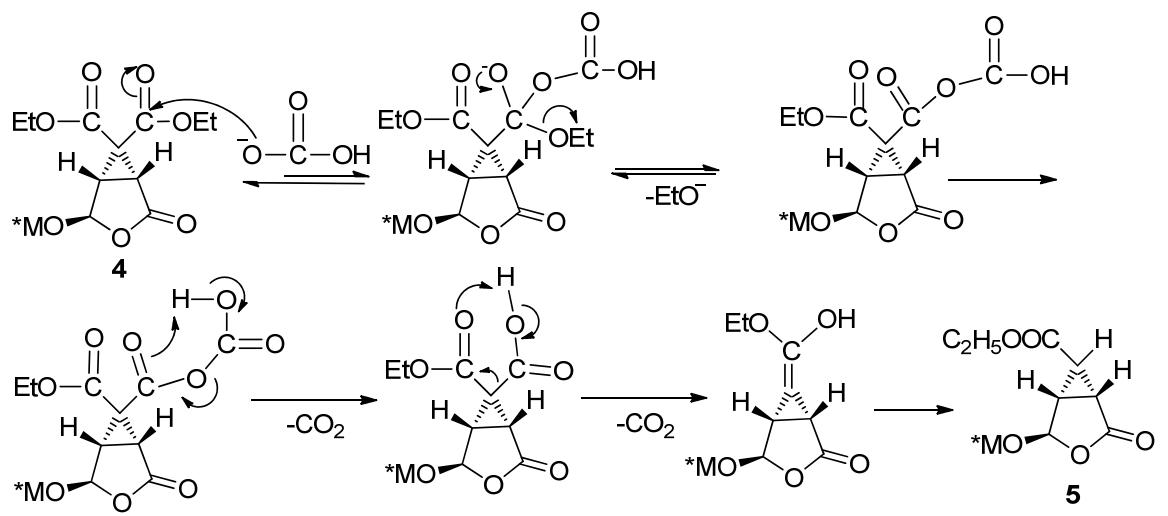

图式 4 化合物 5 形成的反应机理

Scheme 4 Proposed mechanism for the formation of compound 5 
9.1379(11) $\AA, c=11.4137(13) \AA, \beta=106.6950(10)^{\circ}, V=$ 2.249.5(5) $\mathrm{nm}^{3}, Z=4, D_{\mathrm{c}}=1.171 \mathrm{Mg} / \mathrm{m}^{3}, M_{\mathrm{r}}=396.47$. $F(000)=856$, 该化合物的分子结构见图 2. 化合物 5 的 $\mathrm{X}$ 射线单晶衍射数据如下: $\mathrm{C}_{18} \mathrm{H}_{28} \mathrm{O}_{5}$, 单斜晶系, $P 2(1)$ 空 间群. 晶胞参数 $a=5.3331(8) \AA, b=16.629(2) \AA, c=$ 10.5282(15) $\AA, \beta=94.719(2)^{\circ}, V=0.9305(2) \mathrm{nm}^{3}, Z=2$, $D_{\mathrm{c}}=1.158 \mathrm{Mg} / \mathrm{m}^{3}, M_{\mathrm{r}}=324.40 . F(000)=352$, 该化合物 的分子结构见图 3 .

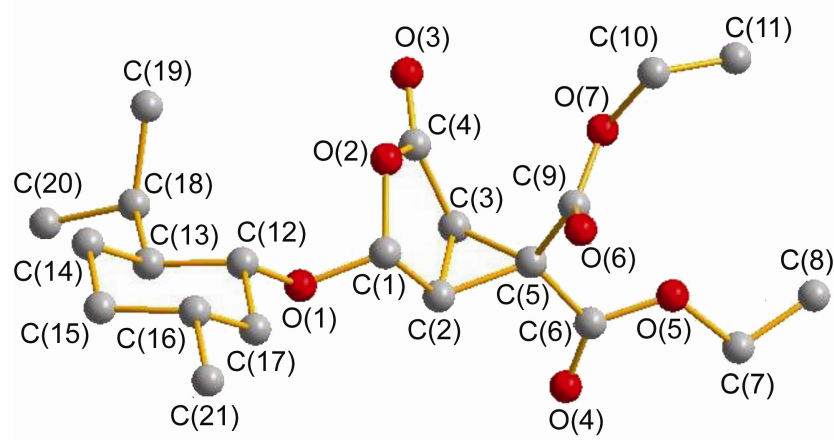

图 2 化合物 $\mathbf{3}$ 的分子结构图

Figure 2 Molecular structure of compound 3

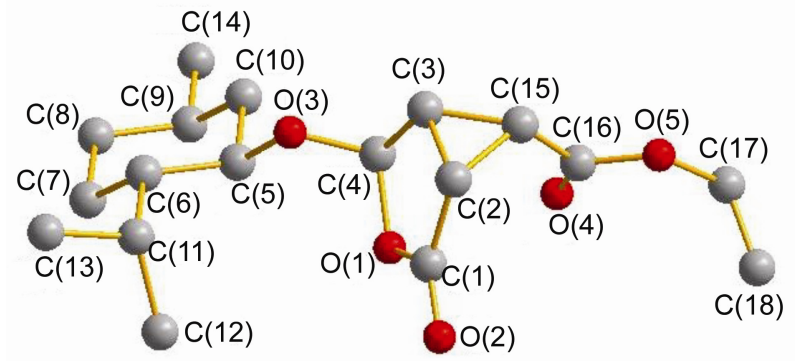

图 3 化合物 $\mathbf{5}$ 的分子结构图

Figure 3 Molecular structure of compound 5

化合物 3 和 5 均为具有三个环的环丙烷并丁内酯的 并环化合物, 其中䓝氧基是一个手性环己烷环, 采用稳 定的椅式构象, 环丙烷环和丁内酯环形成了并环结构, 即双环 [3.1.0]己烷. 在化合物 3 中, 构成环丙烷的键角 分别是 $\mathrm{C}(3)-\mathrm{C}(5)-\mathrm{C}(2)=61.59(15)^{\circ}, \mathrm{C}(2)-\mathrm{C}(3)-$ $\mathrm{C}(5)=59.45(15)^{\circ}, \mathrm{C}(2)-\mathrm{C}(5)-\mathrm{C}(3)=58.95(15)^{\circ}$. 在化 合物 5 中, 构成环丙烷的键角分别是 $C(2)-C(15)-$ $\mathrm{C}(3)=59.3(2)^{\circ}, \mathrm{C}(15)-\mathrm{C}(2)-\mathrm{C}(3)=59.3(2)^{\circ}, \mathrm{C}(2)-$ $\mathrm{C}(3)-\mathrm{C}(15)=61.4(2)^{\circ}$, 均接近 $60^{\circ}$, 与理论值相吻合. 化合物 3 和 5 中的丁内酯结构单元, 构成环的四个碳原 子组成共平面, 氧原子逻出这个平面, 均形成信封式构 象.

由单晶结构可知, 䓝氧基和环丙烷分别处于丁内酯 环平面的两侧, 即处于反式, 这是因为丁烯内酯环具有 平面结构, 由于真氧基的位阻作用, 亲核试剂更易于从 位阻小的一侧进攻, 从而得到䓝氧基与环丙烷处于反式
位置的产物.

\section{2 结论}

以糠醛为原料, 经光氧化、缩醛化反应得到了手性 源 5(R)-( $l$-蒀氧基)-2(5H)-呋喃酮，并以其为合成子，在 无水 $\mathrm{K}_{2} \mathrm{CO}_{3}$ 、四丁基溴化铵 (TBAB) 存在下, 以乙腈为溶 剂, 与 $\alpha$-溴代丙二酸二乙酯发生了 Michael 加成/分子内 亲核取代反应，得到了两种手性环丙烷并丁内酯衍生 物. 其结构经 ${ }^{1} \mathrm{H} N \mathrm{NM},{ }^{13} \mathrm{C} \mathrm{NMR}$ 和 $\mathrm{X}$ 射线单晶衍射进 行了确认．该反应原料易得、反应条件温和、后处理简 单. 结合副产物的结构分析了环丙烷类化合物形成的机 理. 本研究为环丙烷/丁内酯衍生物的合成提供了一种 新的合成策略.

\section{3 实验部分}

\section{1 仪器与试剂}

Bruker DPX 400 (400 MHz)型超导核磁共振仪测定, TMS 为内标; Bruker Apex II X 射线单晶衍射仪; XT5A 显微熔点测定仪, 温度计未校正. 试剂均为分析纯. 固 体试剂直接应用，甲醇、乙醚、乙腈等溶剂按常规方法 干燥重蒸处理.

\section{$3.25(R)-(I$-真氧基)-2(5H)-呋喃酮(1)的合成 \\ 化合物 1 按文献[11]方法合成.}

\section{$3.3 \alpha$-溴代丙二酸二乙酯的合成}

冰浴下, 于 $100 \mathrm{~mL}$ 的三颈烧瓶中, 加入丙二酸二 乙酯 $8.008 \mathrm{~g}(50 \mathrm{mmol}) 、$ 无水乙醚 $50 \mathrm{~mL}$, 边搅拌边滴 加 $8.109 \mathrm{~g}(50 \mathrm{mmol})$ 溴素, 控制滴加温度在 $0 \sim 5{ }^{\circ} \mathrm{C}$. 滴 加完毕后，撤去冰浴，室温搅拌 $12 \mathrm{~h}$ 后停止反应，依次 用蒸馏水、 $\mathrm{NaHCO}_{3}$ 稀溶液洗涤, 水相再用乙酸乙酯一石 油醚混合溶剂萃取, 加入无水硫酸镁干燥, 过滤, 滤液 经减压蒸馏得淡黄色液体状 $\alpha$-溴代丙二酸二乙酯 $13.109 \mathrm{~g}$, 产率 91\%. ${ }^{1} \mathrm{H}$ NMR (400 $\left.\mathrm{MHz}, \mathrm{CDCl}_{3}\right) \delta: 4.84$ (s, 1H), 4.29 (q, $J=7.2 \mathrm{~Hz}, 4 \mathrm{H}), 1.31$ (t, $J=7.2 \mathrm{~Hz}, 6 \mathrm{H})$.

\section{4 化合物 3,5 及 6 的合成}

将 $1.896 \mathrm{~g}(8 \mathrm{mmol})$ 溴代丙二酸二乙酯加到含有

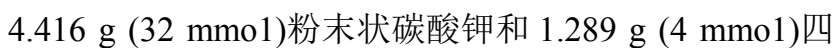
正丁基溴化铵 $(\mathrm{TBAB})$ 的 $30 \mathrm{~mL}$ 乙腈悬浮液中, 搅拌 20 $\min$ 后, 加入 $0.954 \mathrm{~g}(4 \mathrm{mmol})$ 化合物 $5(R)-(l$-䓝氧 基)-2(5H)-呋喃酮, 油浴加热到 $80{ }^{\circ} \mathrm{C}$ 反应，薄层色谱跟 踪反应，待手性源 $5(R)$-( $l$-蓝氧基)-2(5H)-呋喃酮(1)基本 消失后，终止反应，过滤，用乙酸乙酯洗涤固体，将滤 液减压蒸发后得到粗品, 然后经硅胶柱层析分离, 得产 物 3,5 和 6 .

(1R,4S,5R)-4-( $l$-蒀氧基)-6,6-二乙氧羰基-3-氧杂双 
环[3.1.0]己烷-2-酮(3): 白色蜡状物, 产率 34.7\%. m.p. $81.9 \sim 83.0{ }^{\circ} \mathrm{C} ;[\alpha]_{\mathrm{D}}^{20}-46.4\left(\mathrm{c} 0.967, \mathrm{CHCl}_{3}\right) ;{ }^{1} \mathrm{H}$ NMR $\left(400 \mathrm{MHz}, \mathrm{CDCl}_{3}\right) \delta: 5.60(\mathrm{~s}, 1 \mathrm{H}), 4.19 \sim 4.28(\mathrm{~m}, 4 \mathrm{H})$, $3.54(\mathrm{td}, J=8.0,4.0 \mathrm{~Hz}, 1 \mathrm{H}), 2.83 \sim 2.87(\mathrm{~m}, 2 \mathrm{H}), 2.08 \sim$ $2.10(\mathrm{~m}, 2 \mathrm{H}), 1.64 \sim 1.67(\mathrm{~m}, 2 \mathrm{H}), 1.36 \sim 1.38(\mathrm{~m}, 1 \mathrm{H})$, $1.28(\mathrm{t}, J=8.0 \mathrm{~Hz}, 6 \mathrm{H}), 1.21 \sim 1.33(\mathrm{~m}, 1 \mathrm{H}), 0.87 \sim 1.04$ (m, 3H ), $0.93(\mathrm{~d}, J=8.0 \mathrm{~Hz}, 3 \mathrm{H}), 0.88(\mathrm{~d}, J=8.0 \mathrm{~Hz}, 3 \mathrm{H})$, $0.77(\mathrm{~d}, J=8.0 \mathrm{~Hz}, 3 \mathrm{H}) ;{ }^{13} \mathrm{C}$ NMR $\left(100 \mathrm{MHz}, \mathrm{CDCl}_{3}\right) \delta$ : 170.1, 166.1, 163.8, 98.4, 78.1, 63.1, 62.8, 47.8, 40.2, 38.3, $34.7,34.3,31.5,30.0,25.5,23.2,22.3,21.0,15.7,14.1$, 13.8. Anal. calcd for $\mathrm{C}_{21} \mathrm{H}_{32} \mathrm{O}_{7}$ : C 63.60, H 8.08; found $\mathrm{C}$ 63.47, H 8.15 .

$(1 R, 4 R, 5 R, 6 S)-4$ - $(l$-䓝氧基)-6-乙氧羰基-3-氧杂双环 [3.1.0]己烷-2-酮(5): 白色固体, 产率 43.6\%. m.p. 87.1 $89.1{ }^{\circ} \mathrm{C} ;[\alpha]_{\mathrm{D}}^{20}-90.0\left(c\right.$ 1.01, $\left.\mathrm{CHCl}_{3}\right) ;{ }^{1} \mathrm{H}$ NMR $(400$ $\left.\mathrm{MHz}, \mathrm{CDCl}_{3}\right) \delta: 5.64(\mathrm{~s}, 1 \mathrm{H}), 4.16 \sim 4.22(\mathrm{~m}, 2 \mathrm{H}), 3.55 \sim$ $3.62(\mathrm{~m}, 1 \mathrm{H}), 2.53 \sim 2.56(\mathrm{~m}, 1 \mathrm{H}), 2.46 \sim 2.49(\mathrm{~m}, 1 \mathrm{H})$, $2.27 \sim 2.31(\mathrm{~m}, 1 \mathrm{H}), 2.09 \sim 2.15(\mathrm{~m}, 2 \mathrm{H}), 1.65 \sim 1.67(\mathrm{~m}$, $2 \mathrm{H}), 1.31 \sim 1.42(\mathrm{~m}, 1 \mathrm{H}), 1.27(\mathrm{t}, J=8 \mathrm{~Hz}, 3 \mathrm{H}), 1.21 \sim$ $1.29(\mathrm{~m}, 1 \mathrm{H}), 0.90 \sim 1.03(\mathrm{~m}, 3 \mathrm{H}), 0.93(\mathrm{~d}, J=7.2 \mathrm{~Hz}$, $3 \mathrm{H}), 0.88(\mathrm{~d}, J=7.2 \mathrm{~Hz}, 3 \mathrm{H}), 0.80(\mathrm{~d}, J=7.2 \mathrm{~Hz}, 3 \mathrm{H}) ;{ }^{13} \mathrm{C}$ NMR (100 MHz, $\left.\mathrm{CDCl}_{3}\right) \delta: 171.4,167.3,98.0,77.8,62.0$, $47.9,40.3,34.4,31.5,29.7,25.6,25.2,23.8,23.3,22.3$, 21.0, 15.8, 14.2. Anal. calcd for $\mathrm{C}_{18} \mathrm{H}_{28} \mathrm{O}_{5}: \mathrm{C} 66.63, \mathrm{H}$ 8.64; found C 66.71, H 8.52.

$(2 R, 3 R)-2$-溴-2-[5'-( $l$-䓝氧基)-2'-氧代]四氢呋喃-3'基乙酸乙酯(6): 白色固体, 产率 3\%. $[\alpha]_{\mathrm{D}}^{20}-26.4(c$ 0.4, $\left.\mathrm{CHCl}_{3}\right) ;{ }^{1} \mathrm{H}$ NMR (400 MHz, $\left.\mathrm{CDCl}_{3}\right) \delta: 5.51(\mathrm{~s}, 1 \mathrm{H})$, $4.23 \sim 4.26(\mathrm{~m}, 1 \mathrm{H}) 4.18(\mathrm{q}, J=6.8 \mathrm{~Hz}, 2 \mathrm{H}), 3.54 \sim 3.58$ $(\mathrm{m}, 1 \mathrm{H}), 2.61 \sim 2.63(\mathrm{~m}, 1 \mathrm{H}), 2.66 \sim 2.68(\mathrm{~m}, 1 \mathrm{H}), 2.01 \sim$ $2.15(\mathrm{~m}, 2 \mathrm{H}), 1.90 \sim 1.95(\mathrm{~m}, 1 \mathrm{H}), 1.62 \sim 1.70(\mathrm{~m}, 2 \mathrm{H})$, $1.28(\mathrm{t}, J=6.8 \mathrm{~Hz}, 3 \mathrm{H}), 1,20 \sim 1.45(\mathrm{~m}, 2 \mathrm{H}), 0.80 \sim 1.03$ (m, 3H), $0.93(\mathrm{~d}, J=7.2 \mathrm{~Hz}, 3 \mathrm{H}), 0.89$ (d, $J=7.2 \mathrm{~Hz}, 3 \mathrm{H})$, $0.78(\mathrm{~d}, J=6.8 \mathrm{~Hz}, 3 \mathrm{H}) ;{ }^{13} \mathrm{C}$ NMR $\left(100 \mathrm{MHz}, \mathrm{CDCl}_{3}\right) \delta$ : 172.4, 168.7 99.4,77.8, 61.9, 47.8, 40.2, 34.3, 31.5, 30.2, 25.5, 25.1, 24.7, 23.2, 22.3, 21.0, 15.7, 14.0. Anal. calcd for $\mathrm{C}_{18} \mathrm{H}_{29} \mathrm{BrO}_{5}$ : C 53.45, H 7.18; found C 53.28, H 7.12.

\subsection{3 和 5 单晶结构的测定}

经柱层析分离纯化的产物 $\mathbf{3}$ 初为一白色粘稠物质, 放置形成为白色蜡状物, 取少量乙酸乙酯溶解, 待其完 全溶解后, 加入乙醚, 放置挥发, $2 \mathrm{~d}$ 后得无色柱状晶体. 产物 5 则溶解在体积比 $1: 4$ 的乙酸乙酯/石油醚的混合 溶剂中, 室温静置挥发, 得到白色针状晶体.

选取大小合适的化合物 $3(0.32 \mathrm{~mm} \times 0.25 \mathrm{~mm} \times$ $0.17 \mathrm{~mm})$ 及 $5(0.41 \mathrm{~mm} \times 0.38 \mathrm{~mm} \times 0.18 \mathrm{~mm})$ 的晶体,
用 Bruker Smart Apex 衍射仪进行结构测试. 采用经石墨 单色器单色化的 Mo $\mathrm{K} \alpha$ 射线 $(\lambda=0.071073 \mathrm{~nm})$, 以 $\theta-\omega$ 扫描方式, 在 $291(2) \mathrm{K}$ 温度下, $2.34^{\circ}<\theta<25.50^{\circ}$ 范围内, 分别收集到化合物 3 的 8630 个衍射数据 $R(\mathrm{int})=0.0181$, 其中可观测的独立衍射点 4146 个; 化合物 5 的 6457 个 衍射数据 $R(\mathrm{int})=0.0217$, 其中可观测的独立衍射点 3404 个. 全部衍射数据经 LP 因子和经验吸收校正后, 由直接法初步解出各原子位置坐标, 继而经差值 Fourier 合成及最小二乘法修正和各向异性温度因子修 正. 化合物 3 的最终结构偏差因子 $R_{1}=0.0446, w R_{2}=$ $0.1175,[I>2 \sigma(I)]$, 残余电子云密度最高为 $0.215 \mathrm{e} / \AA^{3}$, 最低为 $-0.165 \mathrm{e} / \AA^{3}$. 化合物 5 的最终结构偏差因子 $R_{1}=0.0484, w R_{2}=0.1170,[I>2 \sigma(I)]$, 残余电子云密度最 高为 $0.211 \mathrm{e} / \AA^{3}$, 最低为 $-0.165 \mathrm{e} / \AA^{3}$. 所有氢原子均通 过理论加氢得到. 全部计算工作均在 $\mathrm{PC}$ 机上用 SHELXTL-97 程序包完成. 晶体结构数据存于英国剑桥 数据中心, 其中化合物 $\mathbf{3}$ 的 CCDC 号为 967509 , 化合物 5 的 CCDC 号为 1448119 .

辅助材料(Supporting Information) 化合物 3, 5, 6 的 ${ }^{1} \mathrm{H}$ NMR 和 ${ }^{13} \mathrm{C}$ NMR 谱图. 这些材料可以免费从本刊网 站(http://sioc-journal.cn/)上下载.

\section{References}

[1] Nagao, Y.; Wei, W. M.; Ochiai, M.; Shiro, M. J. Org. Chem. 1989. $54,5211$.

[2] Thurston, L. S.; Imakyura, Y.; Haruna, M. J. Med. Chem. 1989, 32, 604.

[3] Itoh, T.; Chika, J.; Takagi, Y.; Nishiyama, S. J. Org. Chem. 1993, $58,5717$.

[4] Kitson, R. R. A.; Millemaggi, A.; Taylor, R. J. K. Angew. Chem., Int. Ed. 2009, 48, 9426.

[5] Lu, X.; Zhang, Q. Pure Appl. Chem. 2001, 73, 247.

[6] Hang, L.-L.; Xu, M.-H.; Lin, G.-Q. J. Org. Chem. 2005, 70, 529

[7] Yanagisawa, A.; Kushihara, N.; Yoshida, K. Org. Lett. 2011, 13, 1576.

[8] Zeller, M. A.; Riener, M.; Nicewicz, D. A. Org. Lett. 2014, 16, 4810.

[9] Classen, T.; Korpak, M.; Schoelzel, M.; Pietruszka, J. ACS Catal. 2014, 4, 1321.

[10] Feringa, B. L.; de Lange, B.; de Jong, J. C. J. Org. Chem. 1989, 54, 2471

[11] Huang, H.; Chen, Q. Tetrahedron: Asymmetry 1999, 10, 1295.

[12] De Jong, J. C.; Van Bolhuis, F.; Feringa, B. L. Tetrahedron: Asymmetry 1991, 2, 1247.

[13] Curtius, F.; Scharf, H. Tetrahedron: Asymmetry 1996, 7, 2957.

[14] Restullet, A.; Alibs, R.; de March, P.; Figueredo, M.; Font, J. Org. Lett. 2007, 9, 2826.

[15] Möller, T.; Wonneberger, P.; Kretzschmar, N.; Hey-Hawkins, E. Chem. Commun. 2014, 50, 5826.

[16] Hang, H.; Qi, C.; Tian, Z. Chin. J. Org. Chem. 2005, 25, 239 (in Chinese).

(黄海波，齐传民，田作林，有机化学， 2005, 25, 239.) 
[17] Song, X.; Li, J.; Wang, Z.; Mo, Y. Chin. J. Org. Chem. 2010, 30, 648 (in Chinese).

(宋秀美, 李建晓, 汪朝阳, 莫阳青, 有机化学, 2010, 30, 648.)

[18] He, L.; Liu, Y.; Zhang, W.; Li, M.; Chen, Q. Tetrahedron 2005, 61, 8505.

[19] Sreedharan, D. T.; Clive, D. L. Org. Biomol. Chem. 2013, 11, 3128.

[20] Wei, M. X.; Gao, X. H.; Zhang, H.; Li, X. Q. Chin. J. Org. Chem. 2015, 35, 439 (in Chinese).

(魏梦雪, 高晓慧, 张和, 李学强, 有机化学, 2015, 35, 439.)

[21] Lebel, H.; Marcoux, J. F.; Molinaro. C. Chem. Rev. 2003, 103, 977.

[22] Chen, D. Y. K.; Pouwer, R. H.; Richard, J. A. Chem. Soc. Rev. 2012, 41, 4631.

[23] Villar, I. S.; Gradillas, A.; Domínguez, G.; Pérez-Castells, J. Tetrahedron Lett. 2010, 51, 3095.

[24] Luo, J.; Wu, B.; Chen, M. W.; Jiang, G. F.; Zhou, Y. G. Org. Lett, 2014, 16, 2578.
[25] Wang, J. P.; Chen, Q. H. Chin. J. Org. Chem. 2001, 21, 728 (in Chinese).

(王建平，陈庆华，有机化学, 2001, 21, 728.)

[26] Fu, Y.; Fan, X.; Wang, J.; Kang, H.; Chen, Q. Chin. Sci. Bull. 2003, $48,323$.

[27] Guo, J. B.; Zhang, X. Y.; Chen, Q. H. Acta Chim. Sinica 2006, 64, 2008 (in Chinese). (郭金波, 张淅芸, 陈庆华，化学学报, 2006, 64, 2008.)

[28] Yu, Z. L.; Hu, S. Q.; Li, S. L.; Fu, Y. Q. Chin. J. Org. Chem. 2008, 28, 1119 (in Chinese). (郁兆莲, 胡少强, 李森兰, 傅玉琴, 有机化学, 2008, 28, 1119.)

[29] Maestro, M. C.; Barquilla, M. C.; Martin, M. R. Tetrahedron: Asymmetry 1999, 10, 3593.

[30] Oikawa, M.; Sugeno, Y.; Ishikawa, Y.; Tukada, H. Synlett 2013, 24, 886. 